\title{
Estudio de la eficacia del sellado con taurolidina y citrato $4 \%$ del catéter para hemodiálisis en la prevención de infección y trombosis
}

\author{
M $^{a}$ del Rocío González Martínez, $\mathbf{M}^{\mathrm{a}}$ Dolores Ojeda Ramírez, Ana $\mathbf{M}^{\mathrm{a}}$ García Pérez, $\mathbf{M}^{\mathrm{a}}$ del Carmen Redondo \\ Simón, Inmaculada Caro Rodríguez, $\mathbf{M}^{\mathrm{a}}$ Concepción Huerga García, Marta Gómez Cambronero, $\mathbf{M}^{\mathrm{a}}$ del Carmen \\ Molina Álvarez, Sonia García Hita, Rocío Fernández Palenzuela, Yolanda Canovas Padilla \\ Enfermeras en la Unidad de Hemodiálisis del Hospital de Poniente. Almería
}

\begin{abstract}
Resumen
El uso de catéteres centrales ha aumentando de forma importante a pesar de su mayor morbi-mortalidad en comparación con las FAV. Las principales complicaciones son trombosis, bacteriemia-sepsis y limitación de la eficacia dialítica disminuyendo la calidad de vida de los pacientes y elevando el coste sanitario.

En nuestra unidad, el sellado se realizaba con heparina sódica al 5\% y actualmente utilizamos TauroLock ${ }^{\mathrm{TM}}$ Hep 500®, compuesta de (ciclo)-taurolidina (agente antimicrobiano biocompatible), citrato al $4 \%$ y heparina $500 \mathrm{UI} / \mathrm{ml}$.
\end{abstract}

Objetivo: Comprobar la eficacia del Taurolock para reducir la incidencia de colonización intraluminal y trombosis al compararla con heparina sódica al $5 \%$.

Material y método: Realizamos un estudio observacional longitudinal de cohorte y analizamos colonización intraluminal, trombosis, biofilm y uso de antibioterapia. Resultados: Observamos mejoría en la permeabilidad del catéter, disminución del uso de urokinasa, reducción de sesiones en unipunción y del uso de antibiótico.

Conclusiones: Nuestro estudio muestra que el sellado del catéter para hemodiálisis con una solución que contiene taurolidina como antimicrobiano puede reducir significativamente la incidencia de sepsis relacionada con el catéter. La taurolidina parece se efectiva y segura y no conlleva riesgos ni efectos secundarios. La adicción de $\mathbf{5 0 0}$ ui de heparina a la solución de sellado junto con citrato al $4 \%$ ha disminuido los eventos trombóticos reduciendo el uso de fibrilolíticos y el uso de unipunción totalmente, presentando una mejoría de la eficacia dialítica.

$$
\begin{gathered}
\text { Correspondencia: } \\
\mathrm{M}^{\mathrm{a}} \text { del Rocío González Martínez } \\
\text { Calle San Isidro Labrador } \mathrm{n}^{\circ} 14,4^{\circ} 10 \\
\text { El Parador de las Hortichuelas } \\
\text { CP:04720 Almería } \\
\text { E-mail: mrgm1980@hotmail.com }
\end{gathered}
$$

PALABRAS CLAVE:

- CATÉTER CENTRAL

- SELLADO

- INFECCIÓN

- TROMBOSIS

- EFICACIA DIALÍTICA

$\bullet \bullet \bullet \bullet \bullet \bullet \bullet \bullet \bullet \bullet \bullet \bullet \bullet \bullet \bullet \bullet \bullet \bullet \bullet \bullet$

Study of the efficacy of a taurolidine and $4 \%$ citrate lock solution for haemodialysis catheters in the prevention of infection and thrombosis

\section{Abstract}

The use of central catheters has increased significantly despite their greater morbi-mortality compared to AVF. The main complications are thrombosis, bacteriaemiasepsis, and limitation of dialysis efficacy, reducing quality of life for patients and increasing the healthcare cost. In our unit, $5 \%$ heparin sodium was previously used as lock solution and we currently use TauroLock ${ }^{\mathrm{TM}}$-Hep $500 \circledast$, composed of (cyclo)-taurolidine (biocompatible antimicrobial agent) $4 \%$ citrate and heparin $500 \mathrm{UI} / \mathrm{ml}$. Objective: To verify the efficacy of Taurolock in reducing the incidence of intraluminal colonization and thrombosis compared with $5 \%$ heparin sodium.

Material and method: We carried out a longitudinal observational cohort study and we analysed intraluminal colonization, thrombosis, biofilm and use of antibiotherapy.

Results: We observed an improvement in catheter permeability, a reduction in the use of urokinase, reduction in unipuncture sessions and in use of antibiotic.

Conclusions: Our study shows that using a lock solution that contains taurolidine as an antimicrobial agent for haemodialysis catheters can significantly reduce the incidence of catheter-related sepsis. Taurolidine appears to be effective and safe and does not entail risks or side effects. The addition of 500 iu of heparin to the lock solution together with $4 \%$ citrate has reduced thrombotic events by reducing the use of fibrilolytics and elimina- 
ting completely the use of unipuncture, presenting an improvement in dialysis efficacy.

\section{KEY WORDS:}

- CENTRAL CATHETER

- LOCK SOLUTION

- INFECTION

- THROMBOSIS

- DIALYSIS EFFICACY

\section{Introducción}

Las bacteriemias relacionadas con el catéter central para hemodiálisis (HD) es una de las principales complicaciones de los pacientes con Insuficiencia Renal Crónica (IRC) sometidos a esta terapia. En la mayoría de estos pacientes, el catéter suele ser la última alternativa de acceso vascular (AV), tras haber agotado la posibilidad de realizar fístulas arteriovenosas (FAV). En algunos pacientes puede ser el AV de inicio ante la imposibilidad de realizar FAV como consecuencia de otros factores asociados como son la enfermedad psiquiátrica, diabetes con mal patrimonio venoso, insuficiencia cardiaca, etc ${ }^{1}$. Debido a todo esto, el uso de catéteres como AV está aumentando de forma importante en las unidades de diálisis en todo el mundo, a pesar de las muchas evidencias acumuladas respecto a su mayor morbi-mortalidad en comparación con las fístulas, ya sean nativas o protésicas ${ }^{2}$.

La disfunción del catéter limita la eficacia dialítica y tiene como complicaciones asociadas la bacteriemiasepsis y trombosis. La trombosis y colonización intraluminal producida por las bacterias (biofilm), junto con la mayor manipulación del acceso obligada por la disfunción, incrementa la probabilidad de episodios sépticos.

El sellado del catéter con heparina es el método más utilizado y pretende reducir los episodios de disfunción y trombosis, pero su eficacia dista mucho de lo deseable y, además, su uso no está exento de riesgos, principalmente hemorragias o trombopenia inducida por la heparina ${ }^{3}$.

Existen en la literatura muy pocos estudios controlados y aleatorizados que comparen la heparina con otras soluciones de sellado ${ }^{4,5}$.

Actualmente hemos introducido una nueva solución de sellado para catéter que se ha relacionado con eliminación del biofilm y prevención de colonización intralumi- nal y trombosis del catéter para hemodiálisis ${ }^{3}$. Se trata del TauroLock TM_ Hep 500, una solución compuesta de (ciclo)-taurolidina, citrato al $4 \%$ y $500 \mathrm{UI} / \mathrm{ml}$ de heparina (agente antimicrobiano biocompatible y agente anticoagulante respectivamente).

Por tanto lo que pretendemos es comprobar la eficacia de la solución de taurolidina-citrato-heparina (ST) frente a la heparina $5 \%$ planteando los siguientes objetivos:

\section{Objetivo principal:}

Comparar la incidencia de colonización intraluminal y de trombosis entre los dos tipos de sellado.

\section{Objetivos secundarios:}

Valorar la disminución del biofilm (de forma indirecta). Valorar cambios en la eficacia dialítica.

\section{Material y método}

Realizamos un estudio observacional, longitudinal, prospectivo, de cohorte con una muestra de 13 pacientes estables en HD portadores de catéter permanente y temporal.

El periodo de recogida de datos está comprendido desde diciembre de 2010 hasta enero de 2012 (13 meses), obteniéndose un total de 1682 sesiones para su posterior estudio. En este periodo diferenciamos 2 fases. Fase 1: recogida de datos de historia clínica y de enfermería desde diciembre 2010 a mayo 2011 previo a la utilización de nuevo protocolo de cebado (un total de 685 sesiones). Fase 2: de junio 2011 a enero 2012 con la utilización de ST (un total de 997 sesiones). Ver tabla 1.

Tabla 1: Fases del estudio

\begin{tabular}{c|c|c|c|c|c|}
\hline FASE 1 & \multicolumn{5}{|c|}{ FASE 2 } \\
\hline $\begin{array}{c}-6 \\
\text { meses }\end{array}$ & Inicio & 1 mes & 2 meses & 4 meses & 7 meses \\
\hline $\begin{array}{c}\text { Dic } \\
2010\end{array}$ & $\begin{array}{c}\text { Junio } \\
2011\end{array}$ & $\begin{array}{c}\text { Julio } \\
2011\end{array}$ & $\begin{array}{c}\text { Agosto } \\
2011\end{array}$ & $\begin{array}{c}\text { 0ctubre } \\
2011\end{array}$ & $\begin{array}{c}\text { Enero } \\
2012\end{array}$ \\
\hline $\begin{array}{c}\text { Hep. } \\
\text { Sódica } \\
\mathbf{5} \%\end{array}$ & T0 & T1 & T2 & T3 & T4 \\
\hline
\end{tabular}

Para el análisis, se diseño una hoja de recogida de datos, en la que se registraron las siguientes variables: permeabilidad de rama arterial y venosa, ramas inverti- 
das, estado final del dializador y cámaras, flujo efectivo arterial medio real (Qb), presión venosa (PV) media, KT-V DDM (Diálisis Dose Monitor, Nikkiso Co. Japan) y porcentaje de reducción de urea (PRU) DDM, uso de fibrinolíticos (urokinasa), signos de infección (signos de infección pericatéter, fiebre, tiritona intradiálisis, antibioterapia empírica por sospecha de infección de catéter y duración de la misma en días) y germen aislado en rama arterial y venosa.

Se calcularon la media y la desviación estándar (DS). Se estimó un error alfa del 5\% (intervalo de confianza 95\%).Para el análisis de los dato se utilizó el programa informático SPSS 15,0 utilizando la prueba de la t de Student para el análisis de las variables cuantitativas y la prueba de la chi-cuadrado para el análisis de de las variables cualitativas.

\section{Metodología fase 1:}

Hasta ahora la forma de mantener permeable el catéter de hemodiálisis era realizando un lavado por arrastre con $10 \mathrm{ml}$ de suero fisiológico (SF) por cada luz y, posteriormente, sellado con heparina sódica al $5 \%$ (con 2,5 $\mathrm{ml}$ en cada rama), que aunque es un fuerte anticoagulante con efecto sistémico, carece de efecto antibacteriano y no evita la formación del biofilm.

\section{Metodología fase 2:}

En el protocolo de sellado que hemos introducido se mantiene el lavado por arrastre con $10 \mathrm{ml}$ de SF por cada luz y sellado con TauroLock ${ }^{\mathrm{TM}}$ Hep 500 con la cantidad exacta que indique la rama del catéter.

Utilizamos esta solución con una concentración de citrato al $4 \%$ ya que hemos encontrado bibliografía que contraindica mayores concentraciones ( $30 \%$ y $46,7 \%$ ) por riesgos de paro cardiaco temporal ${ }^{6}$ y complicaciones embólicas ${ }^{6,7}$.

\section{Analizamos:}

1. Colonización intraluminal, por medio de signos de infección en orificio de entrada y hemocultivo (HC) de rama arterial y venosa al inicio de cebado ST (T0), 1 mes (T1), 2 meses (T2), 4 meses (T3) y 7 meses (T4). (Tabla 1).

2. Trombosis, por medio de la permeabilidad de cada rama prediálisis, necesidad de urokinasa y sesiones en unipunción.

3. Biofilm, por medio del flujo efectivo arterial (Qb), presión venosa (PV) y Kt/v DDM.

4. Antibioterapia y días de tratamiento.

\section{Resultados}

Se estudian 13 pacientes estables en HD durante una media de 46,6 meses (DS 44,8), con un total de 1682 sesiones; 7 mujeres $(53,8 \%)$; edad media 69 años (DS16, rango 36-87). Los accesos vasculares estudiados son catéter yugular permanente $10(76,9 \%)$; subclavio permanente $1(7,7 \%)$ y femoral transitorio $2(15,4 \%)$.

1. Colonización intraluminal

Al comparar las variables que valoran los signos de infección del orificio de entrada, encontramos una diferencia estadísticamente significativa $(p=0,002)$ entre la fase $1(3,4 \%)$ y la fase $2(1,1 \%)$. (fig.1)

Al valorar la frecuencia de hemocultivos positivos en ambas ramas, se pasó de un $26,7 \%$ al inicio del uso del nuevo protocolo de cebado (TO) a un $0 \%$ al final del periodo estudiado (T4) en la rama arterial, y de un $20 \%$ a un $0 \%$ en la rama venosa, diferencia estadísticamente no significativa $(P=0,223)$. Sin embargo, si comparamos el valor inicial (TO) y final (T4) sí se aprecia una importante disminución de la prevalencia de hemocultivos positivos. (fig 2)

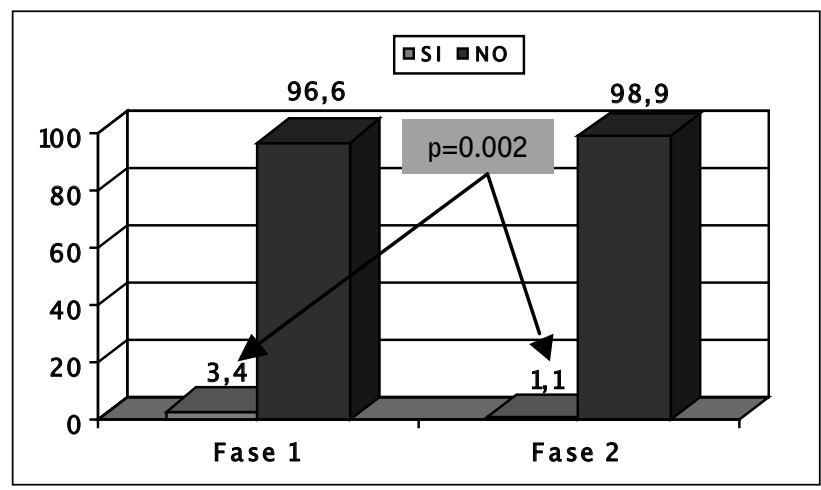

Figura 1. Signos infección pericatéter.

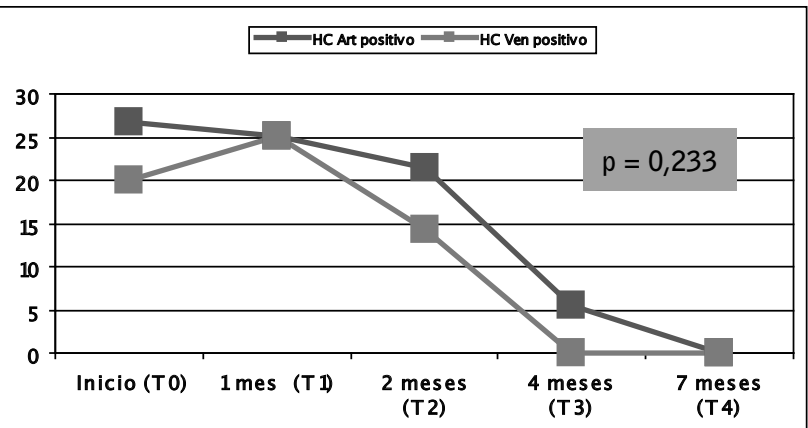

Figura 2. Incidencia HC positivos en fase 2.

\section{Trombosis}

Al comparar la permeabilidad de ambas ramas prediálisis obtuvimos una diferencia significativa $(p<0,0001)$ en la permeabilidad de la rama arterial, 
disminuyendo los casos de no permeabilidad de un $7,4 \%$ en fase 1 a un $0,5 \%$ en la fase 2 (fig.3); en cambio no se apreció mejoría en la permeabilidad de la rama venosa.

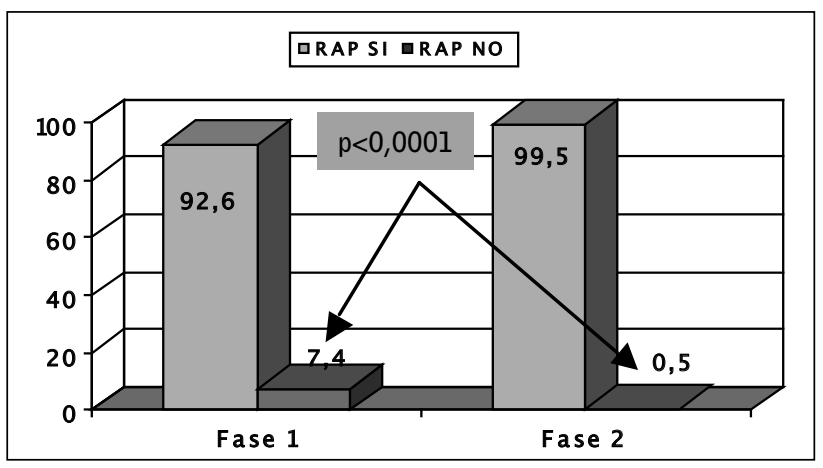

Figura 3. Permeabilidad rama arterial.

Al comparar el uso de urokinasa y las sesiones de unipunción en ambas fases obtuvimos una diferencia significativa en ambos parámetros. En cuanto al uso de urokinasa pasamos de un 6,1\% en fase 1 a $0,1 \%$ en fase 2 ( $p<0,0001$ ). (Fig. 4). En cuanto a la reducción del número de sesiones de unipunción pasamos de un $7,9 \%$ en fase 1 a $0 \%$ en fase 2 ( $p<0,0001$ ). (Fig. 5)

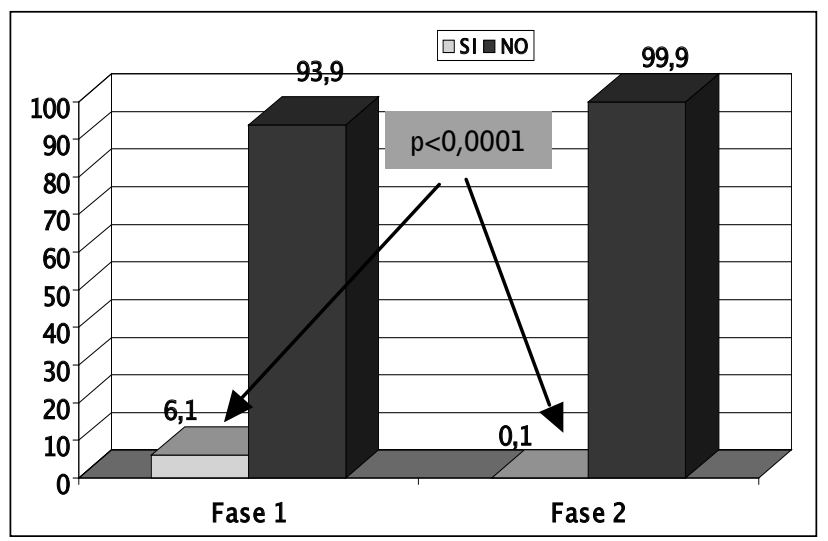

Figura 4. Uso de urokinasa.

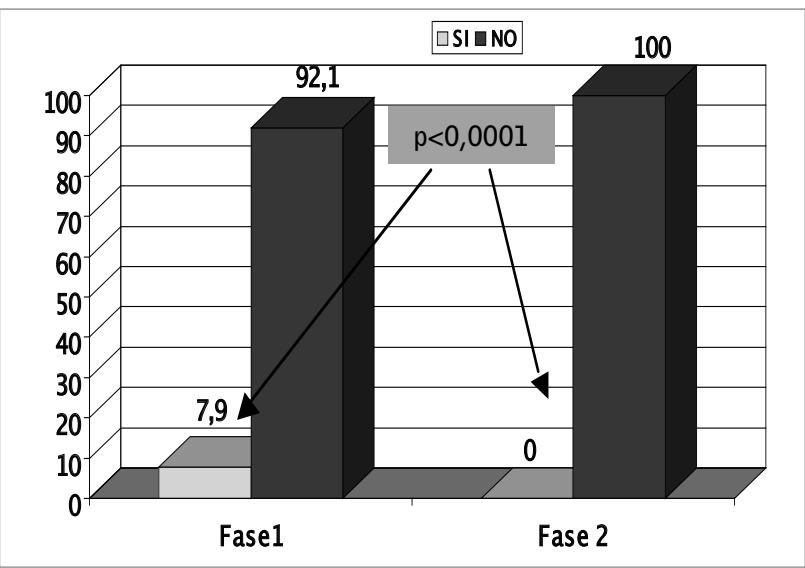

Figura 5. Utilización de Unipunción.

\section{Biofilm}

Comparamos el flujo efectivo arterial que pasó de $314,9 \mathrm{ml} / \mathrm{min}$ en fase 1 a $320,8 \mathrm{ml} / \mathrm{min}$ en fase 2 $(p=0,004)$, la presión venosa pasó de $159,8 \mathrm{mmHg}$ en fase 1 a $161,1 \mathrm{mmHg}$ en fase $2(p=0,338)$ y Kt/v DDM que mejoró su valor de 1,58 en fase 1 a 1,63 en fase 2 $(p=0,002)$ con tendencia a ser estadísticamente significativo lo que nos indica eliminación del biofilm y en consecuencia una mejoría de la eficacia dialítica.

\section{Antibioterapia}

Comparamos la prescripción de tratamiento antibiótico, pasando de 42 sesiones $(6,1 \%)$ en fase 1 a 25 sesiones (2,5\%) en fase 2 ( $p<0,0001$ ). (Fig. 6). Los principales antibióticos utilizados fueron vancomicina, cefazolina y gentamicina.

En cuanto al uso de antibioterapia local se redujo de 14 sesiones $(2 \%)$ en fase 1 a 0 sesiones $(0 \%)$ en fase 2 , siendo los utilizados mupirocina o gentamicina.

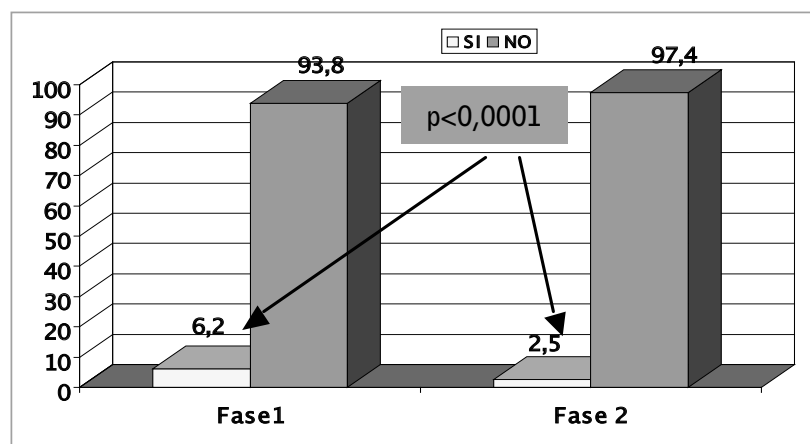

Figura 6. Prescripción de tratamiento antibiótico.

\section{Discusión}

El sellado del cateter con taurolidina-citrato $4 \%$ hep $500 \mathrm{ui} / \mathrm{ml}$ en nuestra unidad ha sido efectivo para reducir la incidencia de infecciones-bacteriemias al comparar con el sellado con heparina $5000 \mathrm{U} / \mathrm{ml}$. Esta conclusión coincide con otros estudios realizados recientemente ${ }^{10,11,12}$, pero no concuerda con la totalidad de los resultados de estos estudios.

En el estudio de Solomon et $\mathrm{a}^{12}$ nos habla del uso de taurolidina-citrato (sin tener asociado heparina) y en sus conclusiones nos habla de una mayor necesidad de uso de tratamiento trombolítico, lo que indica una actividad anticoagulante menos efectiva del citrato al $4 \%$ en comparación con la heparina. Nosotros al tener asociado hep 500 ui/ml a al solución de taurolidina-citrato $4 \%$ tenemos unos resultados de disminución importante de la prevalencia de trombosis, reduciendo totalmente la necesidad de sesiones en unipunción, llegando casi a ser nulo el uso de fibrino- 
líticos y presentando una mejoría significativa en la permeabilidad de las ramas. Si coincide con nuestros resultados el hecho de que asocia el uso de taurolidina-citrato con una disminución de la prevalencia de infecciones en comparación con otros sistemas de sellado de catéteres.

El uso de taurolidina como agente antimicrobiano biocompatible en nuestra unidad ha reducido el uso de antibioticos de forma significativa sin crear resistencias por su uso, resultado que concuerda con otros estudios realizados al respecto $0^{3,10}$. El añadir antibioticos al sellado del catéter puede evitar bacteriemias, sin embargo, su uso puede crear resistencias ${ }^{13}$ como aparece reflejado en muchos estudios. La taurolidina tiene una acción antimicrobiana simple y general y en ensayos in vitro no aparecieron resistencias, siendo por tanto, una solución de sellado efectiva y segura.

Otros estudios ${ }^{5,8}$ muestran como alternativa el sellado con citrato trisódico a altas concentraciones (30\%) ya que presenta una elevada capacidad antimicrobiana sin afectar a la oclusión del catéter. El uso de dichas soluciones se desaconsejó por parte de la FDA (EE.UU) por presentar efectos adversos importantes ${ }^{6,7}$. La concentración de citrato al $4 \%$ si esta recomendada por FDA y se recomienda como una alternativa segura y barata a la heparina ${ }^{9,10}$ pero estas soluciones de sellado muestran una baja actividad antimicrobiana por un lado y un efecto anticoagulante mucho más bajo que el producido por una solucion de heparina.

Nuestra solución de sellado taurolidina-citrato $4 \%$ hep $500 \mathrm{ui} / \mathrm{ml}$ muestra unos resultados prometedores ya que no solo estamos reduciendo la incidencia de infecciones de una forma importante al disminuir la colonización intraluminal, sino que al asociar citrato $4 \%$ con heparina disminuimos los eventos trombóticos de una forma significativa, descendiendo consecuentemente el uso de fibrinolíticos y sus complicaciones potenciales.

Por último exponer que gracias a la nueva solución de sellado en nuestra unidad podemos disminuir costes económicos a medio y largo plazo, reduciendo el uso de antibióticos, fibrinolíticos y la carga de enfermería y del mismo modo, aumentar la calidad de la práctica del tratamiento.

\section{Conclusiones}

Nuestro estudio muestra que el sellado del catéter para hemodiálisis con una solución que contiene taurolidina como antimicrobiano puede reducir significativamente la incidencia de sepsis relacionada con el catéter. La taurolidina parece se efectiva y segura y no conlleva riesgos ni efectos secundarios que sí se han presentado con otras soluciones de sellado antimicrobianas que contienen gentamicina 0 altas concentraciones de citrato. La adicción de 500 ui de heparina a la solución de sellado junto con citrato al 4\% (a una baja concentración recomendada por (a FDA) ha disminuido los eventos trombóticos reduciendo el uso de fibrilolíticos casi en su totalidad en la unidad y el uso de unipunción totalmente, todo ello ha podido influir en el objetivado aumento significativo del $\mathrm{Qb}$ con escasa elevación de la PV e incremento del valor del Kt-v, es decir, en una mejoría de la eficacia dialítica.

En consecuencia, por todo lo anteriormente expuesto, se puede aumentar la vida media del acceso vascular al disminuir la manipulación del mismo, mejorando la calidad y esperanza de vida del paciente, reduciendo la carga de enfermería y el coste económico sanitario.

\section{Limitaciones del estudio}

La muestra de nuestro estudio es baja debido a que en nuestra unidad el porcentaje de pacientes portadores de estos es de un $22 \%$, pero se ha realizado el estudio durante un periodo de 13 meses lo que nos permite valorar un elevado número de casos lo que mejora la fiabilidad del estudio.

Las dificultades encontradas a la hora de realizar este estudio se basan principalmente en el déficit de cuidado relacionado con el acceso vascular por parte del paciente (higiene deficitaria y la "manipulación") no relacionado con déficit de conocimientos. La incidencia de hemocultivos positivos se encuentra sesgada por este motivo.

Recibido: 18 Febrero 2013

Revisado: 14 Marzo 2013

Modificado: 31 Enero 2014

Aceptado: 10 Febrero 2014 


\section{Bibliografía}

1. Martín Chacón et al. Incidencia de bacteriemia en pacientes portadores de catéter permanente tunelizado para hemodiálisis. Rev Soc Esp Enfer Nefrol; 2008; 11 (4): 277/281.

2. Kidney Am J.Vascular Access Cork Group. Clinical Practice guidelines for vascular access, 2006; 48 (suppl 1):S241-S242).

3. Betjes M GM, Van Agteren M. Prevention of dialysis catheter-related sepsis with a citrate-taurolidine containing lock solution, Nephrol Dial Transplant, 2004, 19:1546-1551.

4. Macrae JM el al. Citrate $4 \%$ versus heparin and the reduction of thrombosis study. Clin J Am Soc Nephrol 2008;3: 369-74.

5. Weijmer et al. Randomized, Clinical Trial Comparison of Trisodium Citrate $30 \%$ and Heparin as Catheter-Locking Solution in Hemodialysis Patients. JASN. September.1 2005. Vol. 16 no 9. 2769-77.

6. Punt $C D$, Boer. Paro cardíaco después de la inyección de citrato trisódico concentrado, Clínica de Nefrología 2008, 69 (4), (317-318).

7. Davenport A, Willicombe MK, Vernon K. Las complicaciones embólicas de catéteres venosos centrales de hemodiálisis se utilizan con una solución hipertónica de citrato, American Journal of Kidney Diseases, 2010, 55, 348-351.

8. Weijner c. Superior antimicrobial actibity of trisodium citrate over heparin for catheter lochkng. Nephrol dial Transplant. 2002,17: 2189-95.

9. Lok CE, Appleton D, Bhola C, Khoo B, Richardson RM. Trisodium citrate $4 \%$ an alternative to heparin capping of haemodialysis catheters. Nephrol Dial Transplant 2007; 22: 477-83.

10. Betjes M GM. Prevention of catéter-related boodstream infection in patients on hemodialisis Nat. Rev. Nephrol. 2011, 7: 257-265.

11. Solomon LR et al. Observational study of need for thrombolytic therapy and incidence of bacteremia using taurolidine-citrate-heparin, taurolidine-citrate and heparin catheter locks in patients treated with hemodialysis. Seminars in dialysis. Vol 25, Issue 2. pagea 233-238. March/April 2012.

12. Solomon LR. A randomized double-blind controlled trial of taurolidine-citrate catheter locks for the prevention of bacteremia in patients treated with hemodialysias. American Journal of Kidney Diseases 2010 Vol: 55:1069-1078.

13. Baltrons $A$ et al. Estudio comparativo del sellado de catéteres con citrato trisódico o heparina sódica más gentamicina. Rev Soc Esp Enferm Nefrol v.11 n.2 abr.-jun. 2008. 